\title{
Auswirkungen der COVID-19-Pandemie
}

\author{
Die ZVO-Mitgliedsunternehmen verspüren eine leichte Aufhellung des Geschäftsklimas. \\ Dies ist das Ergebnis der dritten Umfrage zur Betroffenheit der Mitgliedsunternehmen durch \\ die Corona-Krise hinsichtlich der wirtschaftlichen Situation und dem Geschäftsbereich „Messen“.
}

Zwar seien negative Auswirkungen der COVID-19-Pandemie in der Branche spürbar, der Großteil gehe jedoch nicht von langfristigen Folgen für sein Unternehmen aus - so die Ergebnisse der dritten ZVO-Mitgliederbefragung, die zwischen dem 24. September und 15. Oktober durchgeführt wurde. Eine im Vergleich zu den Vorbefragungen im April und Juni optimistischere Einschätzung der Lage. $44 \%$ beurteilen ihre wirtschaftliche Situation aktuell als zufriedenstellend, $31 \%$ als schlecht oder sehr schlecht, $25 \%$ jedoch auch als gut oder sehr gut. Allerdings ist die Situation bei insgesamt $71 \%$ der befragten Unternehmen schlechter beziehungsweise sogar wesentlich schlechter als im Vorjahr.

Die Auftragslage hat sich seit dem Ausbruch der Pandemie im März 2020 gegenüber dem Vorjahr zum Teil deutlich verändert: Etwa $70 \%$ berichten aktuell von einer Reduzierung von bis zu $40 \%$, bei $17 \%$ liegen die Einbußen noch darüber. Knapp $15 \%$ verzeichnen keine Änderung der Auftragslage. Im Vergleich zu den Ergebnissen der beiden vorherigen Befragungen zeichnet sich hier jedoch eine leichte Erholung ab. Für die kommenden zwölf Monate erwarten $38 \%$ keine nennenswerten Veränderungen der wirtschaftlichen Situation, $34 \%$ eine Verbesserung und $28 \%$ eine Verschlechterung.

Die durchschnittliche Kapazitätsauslastung lag im Oktober bei $74 \%$. Bei knapp $60 \%$ der befragten Unternehmen ist kein Personalabbau geplant. Eine etwaige Reduzierung der Beschäftigung wird über- wiegend bei unter $5 \%$ liegen. Nur $40 \%$ der Betriebe haben (noch) Kurzarbeit. Zum Vergleich: Im April waren es noch $65 \%$, im Juni $62 \%$. Die Kurzarbeit betrifft Fertigung und Verwaltung nahezu gleichermaßen. Rund $65 \%$ haben öffentliche Unterstützungsmaßnahmen in Anspruch genommen. Der Anteil an Unternehmen, die Soforthilfe beziehungsweise Kredite beantragt haben, ist im Vergleich zu April und Juni leicht gestiegen auf rund $16 \%$ beziehungsweise $20 \%$.

\section{Krise als Chance begreifen}

Als mögliche langfristige Auswirkungen der Corona-Pandemie sehen die befragten Unternehmen nicht nur negative Aspekte wie eine Reduzierung der Unternehmensgröße, der Umsätze und der Beschäftigung, sondern auch eine Umstellung des Angebots beziehungsweise das Erschließen neuer Märkte, eine beschleunigte Digitalisierung, eine Entspannung auf dem Arbeitsmarkt oder eine Rückverlagerung der Produktion nach Europa. Die Krise kann auch als Chance begriffen werden, aus der die Branche gestärkt herausgeht. Mit einer Normalisierung der Situation rechnen die meisten Unternehmen jedoch erst ab Mitte nächsten Jahres.

\section{Marketing-Instrument „Messen“}

$90 \%$ der befragten Unternehmen halten die Absage der Messe Surface Technology Germany für die richtige Entscheidung. Für sie wäre eine Vorbereitung der Messe nicht $\mathrm{zu}$ leisten gewesen beziehungsweise unter den aktuellen Bedingungen wäre mit zu wenigen Besuchern zu rechnen gewesen. Ein Großteil der Befragten geht davon aus, dass die Corona-Pandemie auch weiterhin Auswirkungen auf das Marketinginstrument „Messen“ haben wird: Für mehr als die Hälfte werden Messegesellschaften umdenken und hybride Formate mit Online-Beteiligungsmöglichkeit anbieten müssen. Für $16 \%$ wäre eine $\mathrm{Zu}$ kunft auch ohne Messen denkbar. $12 \%$ sind dagegen der Meinung, dass Messen wichtiger werden, da der Markt nach der Pandemie die persönliche Begegnung in konzentrierter Form benötige, um das Geschäft neu anzukurbeln. Nur $13 \%$ glauben, dass Messen nach der Pandemie wieder in gewohnter Form durchgeführt werden.

\section{Deutschland bleibt Technologieführer}

Der VDMAOberflächentechnik teilt im Wesentlichen die Einschätzung des ZVO und rechnet mit einem Umsatzrückgang von im Schnitt etwa $20 \%$ im Gesamtjahr 2020. Für 2021 wird das Niveau von 2020 erwartet. 2019 war der Außenhandel laut VDMA Oberflächentechnik noch auf $4 \mathrm{Mrd}$. Euro angewachsen. China führte dabei mit $21 \%$ die Rangliste an und hat damit Deutschland (15\%) in der Außenhandelsstatistik abgelöst. Deutschland bleibt jedoch Technologieführer. Zentrales Thema aller Branchen, Bereiche und Verbände sei die Digitalisierung. // 